\title{
Foot Abnormality Mapping using Statistical Shape Modelling
}

\author{
Kristina STANKOVIĆ*1 Femke DANCKAERS $^{1}$, Brian G. BOOTH ${ }^{1}$, \\ Fien BURG ${ }^{2}$, Saartje DUERINCK ${ }^{2}$, Jan SIJBERS ${ }^{1}$, Toon HUYSMANS ${ }^{1}$ \\ ${ }^{1}$ iMinds - Vision Lab, Dept. of Physics, University of Antwerp, Belgium \\ ${ }^{2}$ RSscan International NV, Paal, Belgium \\ DOI: $10.15221 / 16.070$ http://dx.doi.org/10.15221/16.070
}

\begin{abstract}
About $20 \%$ of the population suffer from disabling foot or ankle pain that require the use of foot orthotics. Traditionally, those foot orthotics are designed manually, but digital procedures are desired to provide a faster, more objective, and more reliable workflow. In this study, we introduce a method for detecting shape abnormalities in feet for the purposes of pathology diagnosis and orthotic design. The proposed method consists of two phases. In the training phase, a statistical 3D foot model (based on 42 healthy subjects) is built. In the test phase, the landmarks of a new 3D foot scan are compared to the trained model. A landmark is detected as an outlier if it is in the extreme ranges. This testing process is repeated at all landmarks to identify all abnormal foot regions. Preliminary results show that, when testing a foot of a known pathology (hallux valgus, heel spur, foot pronation), we are able to detect abnormal regions accurately. We also examined the effect of using rigid or similarity-based alignment during 3D model building and abnormality detection. We show that our proposed method is a faster and a more objective approach than traditional approaches for abnormality detection of the foot. As such, this method may prove useful in the medical diagnosis of foot pathologies and in automated orthotic design.
\end{abstract}

Keywords: abnormality mapping, shape modelling, foot orthotics, diagnosis

\section{Introduction}

Foot orthotics, such as insoles, custom shoes, and ankle-foot orthotics can provide support, correction, and pain management. Design and production of such orthotics rely on plaster casting and manual correction, both of which are labor-intensive and lead to variable end results. The design of these orthotics relies on the analysis of a person's balance and gait. That analysis is often subjective and time consuming [1]. Therefore a lot of effort has been put in development of new workflow that should remove these disadvantages [2, 5-8].

A digital orthotics workflow [2] consisting of digital patient intake, digital measurement, computeraided analysis, orthotic modeling, and digital fabrication, is a promising alternative with advantages in accuracy, time and costs. A statistical approach in digital orthotics leads to more objective analyses and more consistent orthotic designs, ultimately leading to more reliable products. To achieve a digital orthotic workflow we must first automate the examination and abnormality detection for the foot. The results obtained after automated examination can then guide the design of appropriate shoes.

Most common ways of the automated examination involve plantar pressure imaging and 3D foot scanning. For plantar pressure imaging, techniques like pedobarographic statistical parametric mapping (pSPM) [3] and center of pressure trajectory analysis [4] have been used to capture spatial and temporal gait information respectively. These techniques however do not capture the complex 3D shape of the foot. As a result a number of surface scanning systems have become available for acquiring the 3D foot shape that can be viewed and analyzed on a computer. In the paper of Jee et al. [5] they propose the profiling stature and body from measurements based on 3D foot scans. The study of Price et al. [6] showed the possibility of quantifying the foot morphology of adults who are overweight and obese. Kim et al. [7] suggested a method for the classification of elderly woman's foot type. In the study of Lee et al. [8], foot shape characteristics are investigated from a 3D foot shape database for comparing the gender and ethnic differences. While these techniques perform statistical analysis and classification of 3D foot scans, they do not identify and map out subject-specific abnormalities.

\footnotetext{
* Kristina.Stankovic@uantwerpen.be
} 
While abnormality mapping techniques are uncommon in the assessment of foot pathologies, they are commonly seen in the field of radiology. Examples of such abnormality mapping techniques include temporal image subtraction (to highlight the evolution of a pathology), neural networks (to learn relationships between the images and a pathology), and image database methods (to find cases with medical images most similar to the test image) [9]. More recently, the STEAM abnormality mapping technique was proposed that highlights abnormalities by comparing to a statistical model of a healthy population [10]. A statistical 3D shape model of the foot is possible solution that should enable a robust and automated process, but also allows more reliable diagnoses of foot pathologies.

In this paper, we present a method for building a 3D statistical foot model that allows us to detect abnormalities of pathological feet. To detect abnormalities we create a 3D healthy foot model that we can compare to the test foot. To create the 3D foot model we establish correspondences between all feet within database. After correspondence establishment, a 3D statistical foot model can be built by aligning the foot shapes and fitting Normal distributions to their vertex locations. We also examine the effect of different alignment procedures during 3D foot model building and abnormality detection (rigid versus similarity). Our experiments on 3D foot database showed that we successfully detect points of the foot surface that are outliers with respect to our healthy foot model and form regions of abnormalities. The proposed method leads to a more objective examination of foot pathologies that takes less time and costs less compared to traditional approaches.

\section{Methods}

The proposed method consists of two phases. In the training phase a 3D foot model is built by registering all 3D foot scans within the database to a common reference surface. The resulted 3D foot model is required in the test phase where we detect outliers and mark them as regions of abnormalities. The flowchart of the proposed method for abnormality detection is illustrated in Fig. 1 and will be explained in detail in following sections.

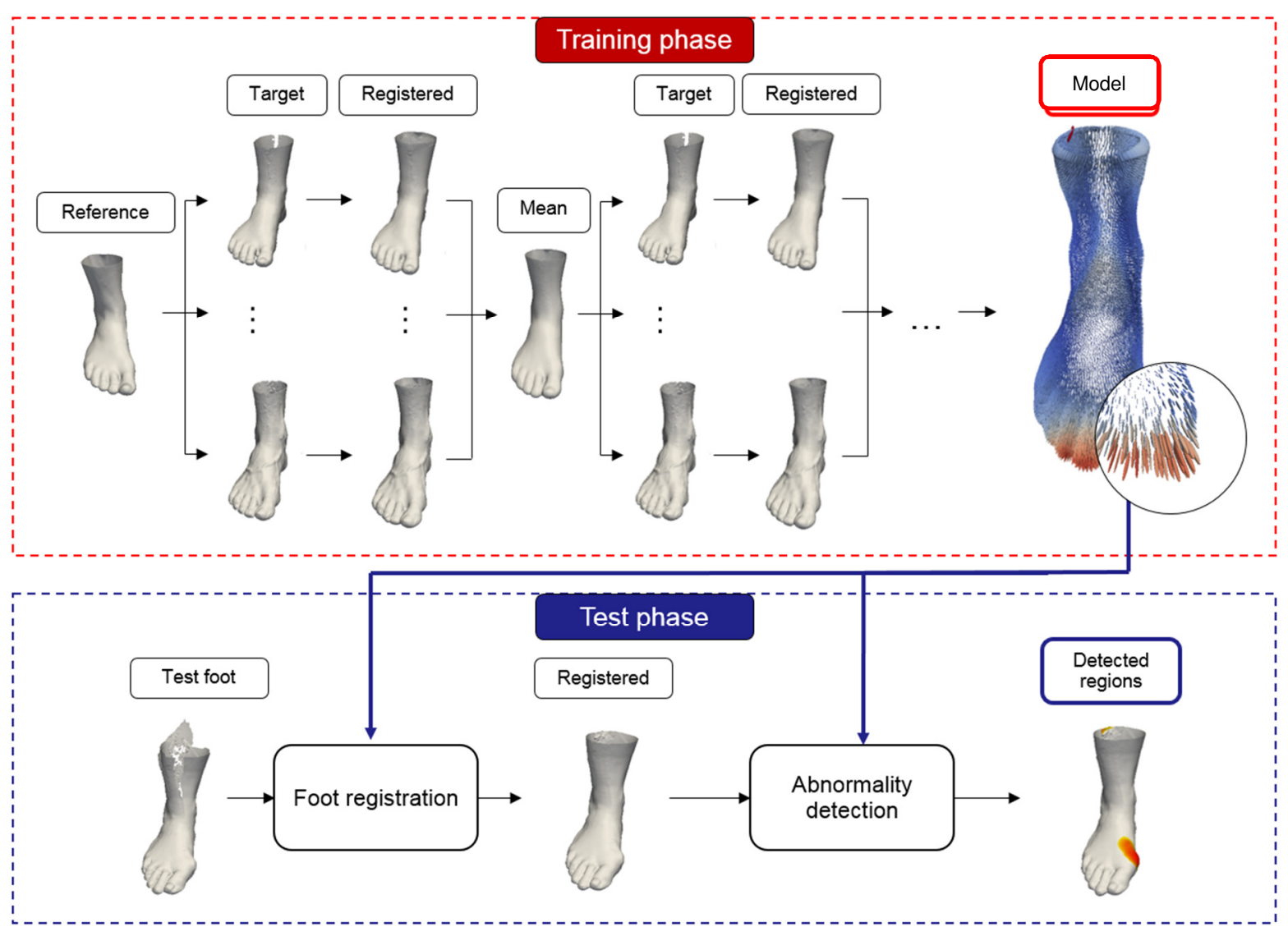

Fig. 1. The abnormality detection framework 


\subsection{Training phase}

From a training set of $3 D$ foot scans, a $3 D$ statistical foot model is built to describe the healthy population. To build the 3D foot model, the first step is to define the correspondence between vertices on the feet. Once the correspondence is established, we can align the feet and create an unbiased 3D foot template. We then build our statistical model in the form of our unbiased 3D foot template.

\subsubsection{Shape correspondence}

Different papers introduce different methods for establishing a correspondence between two 3D shapes [11, 12, 13]. As proposed in [12], we establish correspondence using pair-wise registration with a common reference surface. In this part, a reference foot is registered to a target foot, such that geometric distance between those surfaces becomes minimal while maintaining vertex correspondences. To get good registration the reference foot has been uniformly resampled and made watertight [14]. An example of the foot registration from the reference foot to the target foot is shown in Fig 2.

To obtain the correspondence we first perform a global affine registration. Then, a global affine registration and an elasticity modulated registration are iteratively repeated. During the iterations, the stiffness of the elastic registration gradually decreases which enables the surface to better match the target foot. In the elastic part of the registration, the movement of neighboring vertices is constrained, resulting in similar movements for nearby vertices. The constrained movement is a trade-off of maintaining the shape's integrity and accuracy in vertex correspondence.

Corresponding vertices are identified by casting a ray along the normal from each vertex of the reference foot to the target foot. When the normal of an intersection point is in the same direction (within a tolerance) as the normal of the point on the reference foot, those points can be considered corresponding. An additional restriction for corresponding points is that the normal may not intersect the surface multiple times before reaching the corresponding point. Those points are used to align the feet via a least squares estimation of the transformation.
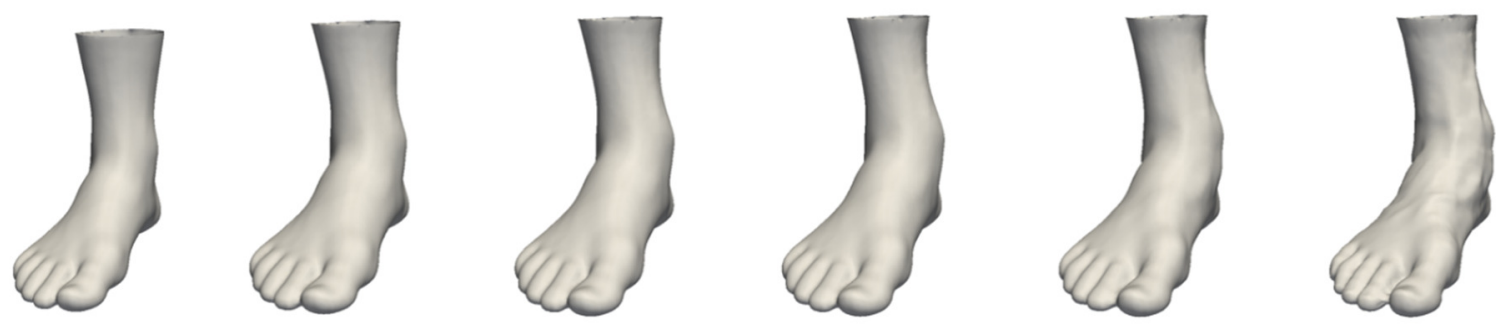

Fig. 2. Example of the foot registration from reference to the target foot (from left to right) through the iterations

\subsubsection{Building an unbiased 3D foot template}

To build a 3D foot template we obtain the correspondences for each foot and align them to a common unbiased space. It is important that all the foot surfaces are superimposed to obtain the appropriate average foot.

Initially a random foot from the database is selected as the reference foot for the pair-wise registration. Next, the reference foot is registered to each foot within the database. From the obtained correspondences, we align the feet using rigid transformations calculated by Procrustes analysis [15]. Once the feet are aligned we average the vertex locations to create the average foot. Because we used rigid transformations to align the feet, the resulting average foot will have an average shape and size, thereby making the average foot unbiased.

We repeat the above process three times to reduce the impact of alignment errors that may occur in the Procrustes analysis. In subsequent iterations, the resulting average 3D foot surface is used as the reference foot. In the final iteration of our procedure, the obtained average 3D foot is declared as an unbiased 3D foot template.

\subsubsection{Building a statistical 3D foot model}

Once we have unbiased 3D foot template we can align all the feet to that template to build a statistical 3D foot model. This model consists of Normal distributions for the locations of each vertex. We fit these Normal distributions using the position of each vertex on the foot surface within database, giving us a mean and 3D covariance for the location of each vertex. 


\subsection{Test phase}

In this phase, we perform T2-tests for abnormality detection or type classification of a new test foot. To that end, we use the 3D foot model obtained in the training phase. First, we obtain a correspondence between the model's mean shape and the test foot using the algorithm described in Section 2.1.1. Then, the test foot is aligned to the mean using the least square alignment. Once the test image and the model are aligned, we perform our T2-tests at each vertex.

\subsubsection{T2-test}

In the T2-test each vertex of the test foot is compared to the Normal distribution of the 3D foot model. An example of a normally distributed vertex is shown on Fig. 3. The T2-test calculates the Mahalanobis distance for each vertex of the test foot using the following formula:

$$
M D(x)=\sqrt{(x-\mu)^{T} \Sigma^{-1}(x-\mu)}
$$

where $x$ a 3D vertex on the test foot, $\mu$ the corresponding vertex on the average foot surface, $\Sigma$ the covariance matrix of the normal distribution for that vertex in the foot model. A vertex on the test foot is detected as an outlier if its $p$-value is smaller than 0.05 . The $p$-value is calculated by using the following formula:

$$
\text { pValue }=1-c d f\left(\chi^{2}, M D(x)\right)
$$

where $c d f$ the cumulative distribution function for the $\chi^{2}$ distribution. Fig. 4 illustrates the probability density function $-p d f$ and cumulative distribution function - $c d f$ of the $\chi^{2}$ distribution with three degrees of freedom, and the $p=0.05$ threshold for outliers detection. All detected outliers together form regions that are geometrically deviating from what was observed in the healthy training population and those regions are considered the result of a pathology or deviating foot type for the test foot. To correct for multiple comparisons, we applied the False Discovery Rate (FDR) procedure. During comparison tests we require FDR with the number of tests equal to the number of vertices of the 3D foot model.

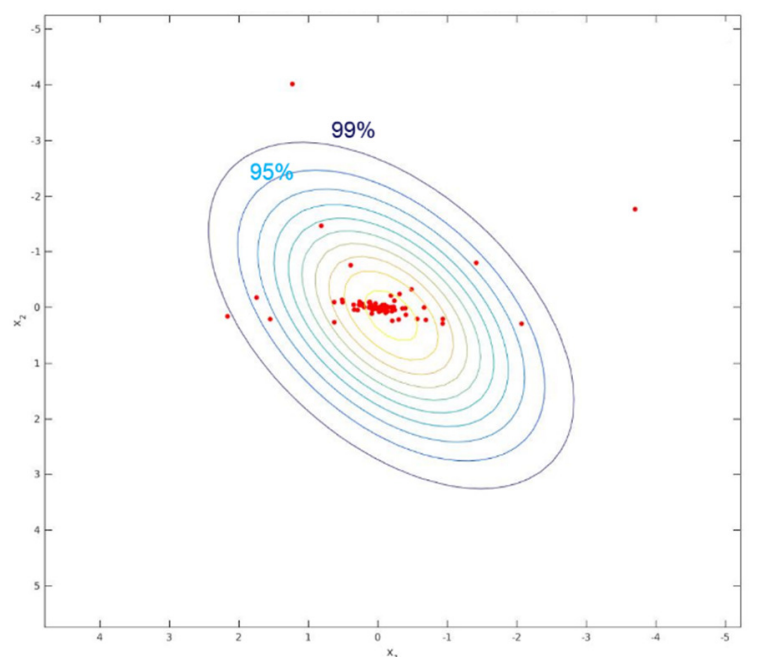

Fig. 3. A Normal distribution of a landmark

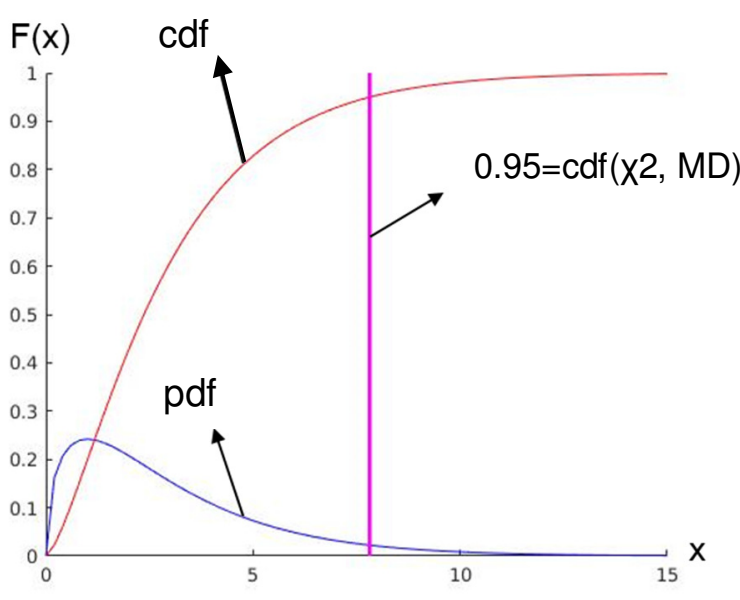

Fig. 4. $X^{2}$ distribution with 3 degrees of freedom

\section{Experiments}

The healthy foot population was selected from a 3D foot scan database which was collected within the framework of the ICON Footwork project [16]. Our database contains 84 3D foot scans of 42 healthy subjects, from which 42 scans are of half loaded (i.e. standing on both feet in balance) right legs and 42 scans are of half loaded left legs. For model creation, we divide this healthy population into two groups: one group with flat feet (9 subjects) and one group with normal foot arches (33 subjects). Separate models are created for both groups.

We performed two experiments to validate our technique. The goal for the first experiment was to build 3D foot models for both healthy subject groups using the technique explained in Section 2.1. The challenge of the second experiment is to detect abnormalities or to classify the type of the selected test foot using our healthy subject models and the abnormality mapping technique described 
in Section 2.2. During these experiments, we have also had different setups in which the datasets were aligned using rigid transformation (as described earlier) or similarity transformations during model building and abnormality detection.

In the first experiment we performed the foot registration within the database. In the first iteration of our procedure, as explained in 2.1.1, a reference foot was selected from the database, uniformly resampled (14,707 points) and made watertight. We performed a minimum of 30 iterations of the shape correspondence algorithm with a decreasing stiffness factor during the iterations (from 100.0 to 10.0). As explained in 2.1.2, the 3D foot template was built in each iteration of our procedure. The obtained 3D foot template is used as a reference foot for the next iteration.

For the second experiment we require a 3D foot model and a new test foot. For the new test foot, twelve 3D foot scans with known pathologies (hallux valgus, heel spur, a foot with pronation) were chosen from a different database in the same Footwork project, so we could confirm that the regions of abnormalities are truly detected. Regions of abnormalities were created from points with $p$-value smaller than 0.05 . In addition the multiple comparison correction is conducted with False Discovery Rate (FDR).

\section{Results}

\subsection{Mean foot model for different foot types}

For a given dataset of 42 healthy subjects, two 3D foot models are built using the proposed methods. During 3D foot model building, similarity or rigid alignment was applied, resulting in a normalized or non-normalized 3D foot model, respectively. The normal arched population model is shown in Fig. 5 and Fig. 6. Identically, normalized and non-normalized 3D flat foot models were created and are shown on Fig. 7 and Fig. 8.
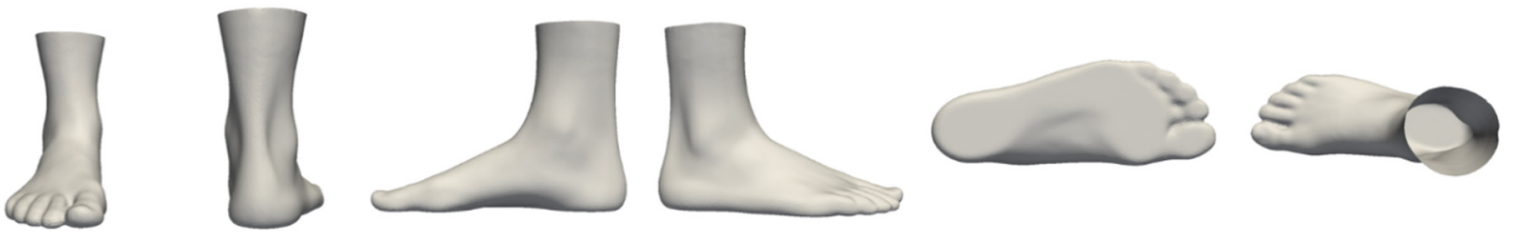

Fig. 5. A normalized 3D foot model (mean foot length $262.2 \mathrm{~mm}$ ) obtained using similarity alignment
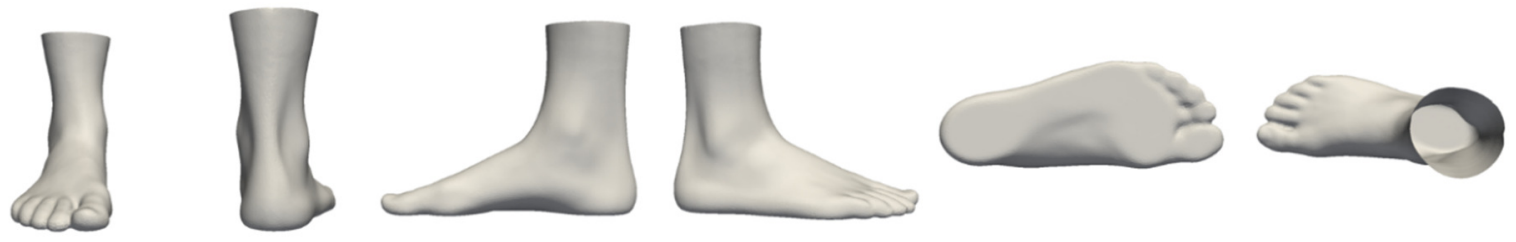

Fig. 6. A non-normalized 3D foot model (mean foot length $262.4 \mathrm{~mm}$ ) obtained using rigid alignment
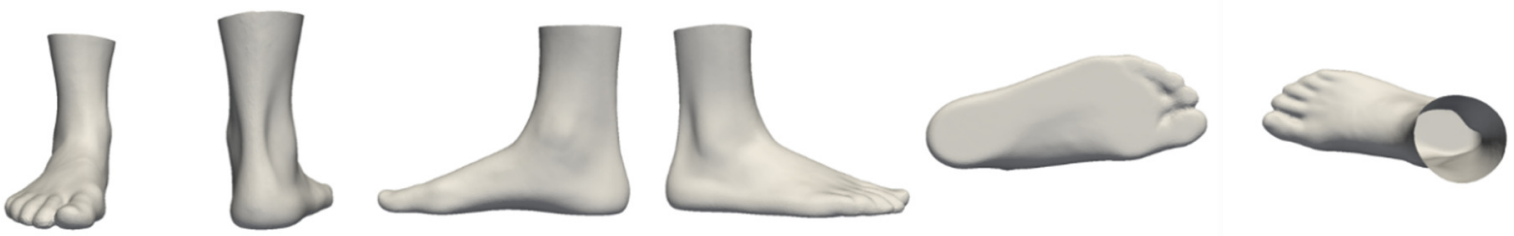

Fig. 7. A normalized 3D flat foot model (mean foot length $264.7 \mathrm{~mm}$ ) obtained using similarity alignment 

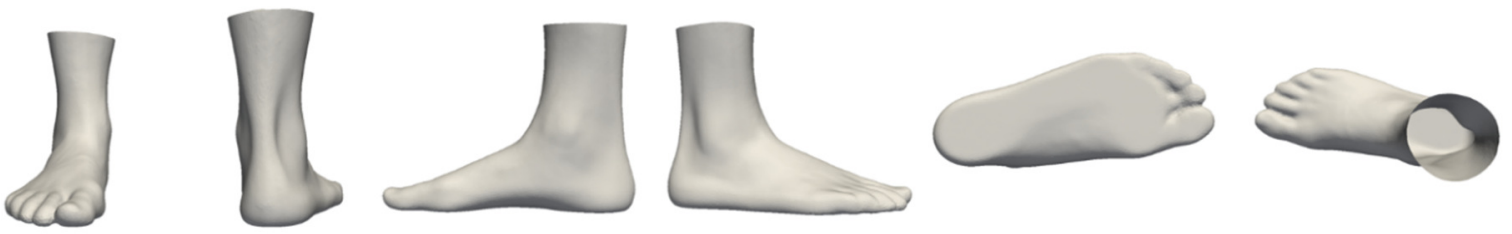

Fig. 8. A non-normalized 3D flat foot model (mean foot length $265.0 \mathrm{~mm}$ ) obtained using rigid alignment

\subsection{Abnormality mapping}

During the test phase, the 12 feet with known pathologies were tested. Here, we show three representative examples of our technique: one from a foot with hallux valgus, one from a foot with a heel spur, and one from a foot with pronation. Abnormality regions were detected in all three cases. Depending on different alignment applied during the 3D model building and abnormality detection, different sizes of detected regions for the same pathology were observed (shown in Table 1). Table 2 shows different sizes of detected regions after applying multiple comparison correction using FDR. Together, points with a $p$-value smaller than 0.05 create regions of abnormalities. Feet with different pathologies (hallux valgus, heel spur, pronation) and detected regions before and after FDR correction are shown in Fig. 9 for non-normalized and normalized test feet respectively.

Table 1. Foot sizes and sizes of detected abnormal regions for different foot pathologies without FDR correction.

\begin{tabular}{|cccc|ccc|} 
& \multicolumn{3}{c|}{ Non-normalized } & \multicolumn{3}{c}{ Normalized } \\
& $\begin{array}{c}\text { Foot } \\
\text { surface } \\
{\left[\mathrm{mm}^{2}\right]}\end{array}$ & $\begin{array}{c}\text { Detected } \\
\text { regions } \\
{\left[\mathrm{mm}^{2}\right]}\end{array}$ & $\begin{array}{c}\text { Detected } \\
\text { regions }[\%]\end{array}$ & $\begin{array}{c}\text { Foot } \\
\text { surface } \\
{\left[\mathrm{mm}^{2}\right]}\end{array}$ & $\begin{array}{c}\text { Detected } \\
\text { regions } \\
{\left[\mathrm{mm}^{2}\right]}\end{array}$ & $\begin{array}{c}\text { Detected } \\
\text { regions } \\
{[\%]}\end{array}$ \\
$\begin{array}{c}\text { Hallux } \\
\text { valgus }\end{array}$ & $82,195.4$ & $2,472.4$ & $\mathbf{3 . 0 0}$ & $87,778.9$ & $3,871.7$ & $\mathbf{4 . 4 1}$ \\
\hline Heel spur & $84,340.6$ & $9,790.1$ & $\mathbf{1 1 . 6 1}$ & $88,758.7$ & $4,582.9$ & $\mathbf{5 . 1 6}$ \\
\hline Pronation & $86,798.3$ & $16,436.0$ & $\mathbf{1 8 . 9 4}$ & $82,583.7$ & $13,850.6$ & $\mathbf{1 6 . 7 7}$ \\
\hline
\end{tabular}

Table 2. Foot sizes and sizes of detected abnormal regions for different foot pathologies with FDR correction.

\begin{tabular}{|cccc|ccc|} 
& \multicolumn{3}{c|}{ Non-normalized } & & & \\
& $\begin{array}{c}\text { Foot } \\
\text { surface } \\
{\left[\mathrm{mm}^{2}\right]}\end{array}$ & $\begin{array}{c}\text { Detected } \\
\text { regions } \\
{\left[\mathrm{mm}^{2}\right]}\end{array}$ & $\begin{array}{c}\text { Detected } \\
\text { regions } \\
{[\%]}\end{array}$ & $\begin{array}{c}\text { Foot } \\
\text { surface } \\
{\left[\mathrm{mm}^{2}\right]}\end{array}$ & $\begin{array}{c}\text { Detected } \\
\text { regions } \\
{\left[\mathrm{mm}^{2}\right]}\end{array}$ & $\begin{array}{c}\text { Detected } \\
\text { regions } \\
{[\%]}\end{array}$ \\
$\begin{array}{c}\text { Hallux } \\
\text { valgus }\end{array}$ & $82,195.4$ & 1727.8 & $\mathbf{2 . 1 0}$ & $87,778.9$ & $2,419.9$ & $\mathbf{2 . 7 6}$ \\
Heel spur & $84,340.6$ & 3445.1 & $\mathbf{4 . 0 8}$ & $88,758.7$ & 172.2 & $\mathbf{0 . 1 9}$ \\
Pronation & $86,798.3$ & 5663.8 & $\mathbf{6 . 5 3}$ & $82,583.7$ & $8,031.3$ & $\mathbf{9 . 7 2}$ \\
\hline
\end{tabular}




\section{Hallux valgus}

uncorrected

FDR correction

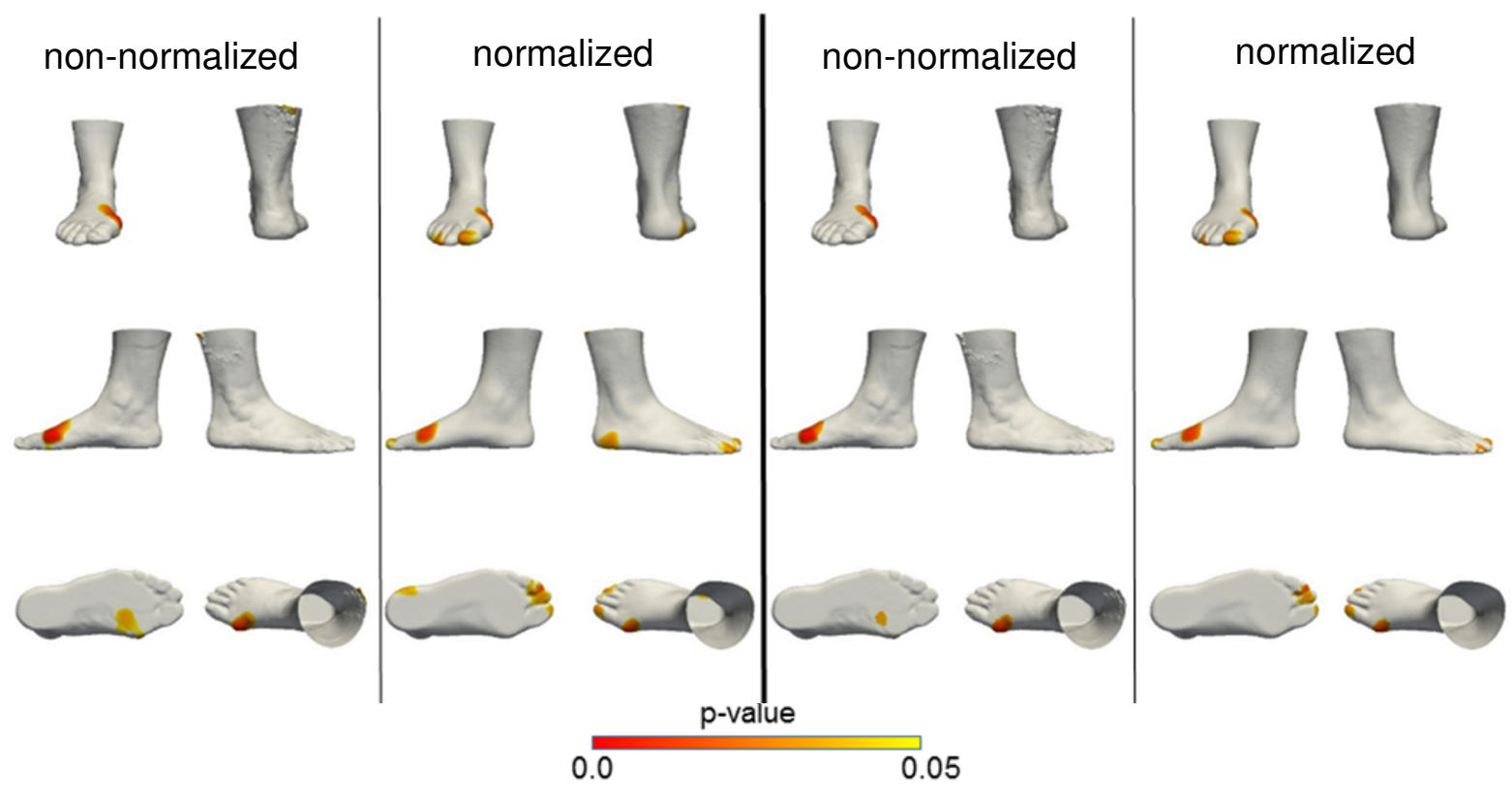

Heel spur

uncorrected

FDR correction

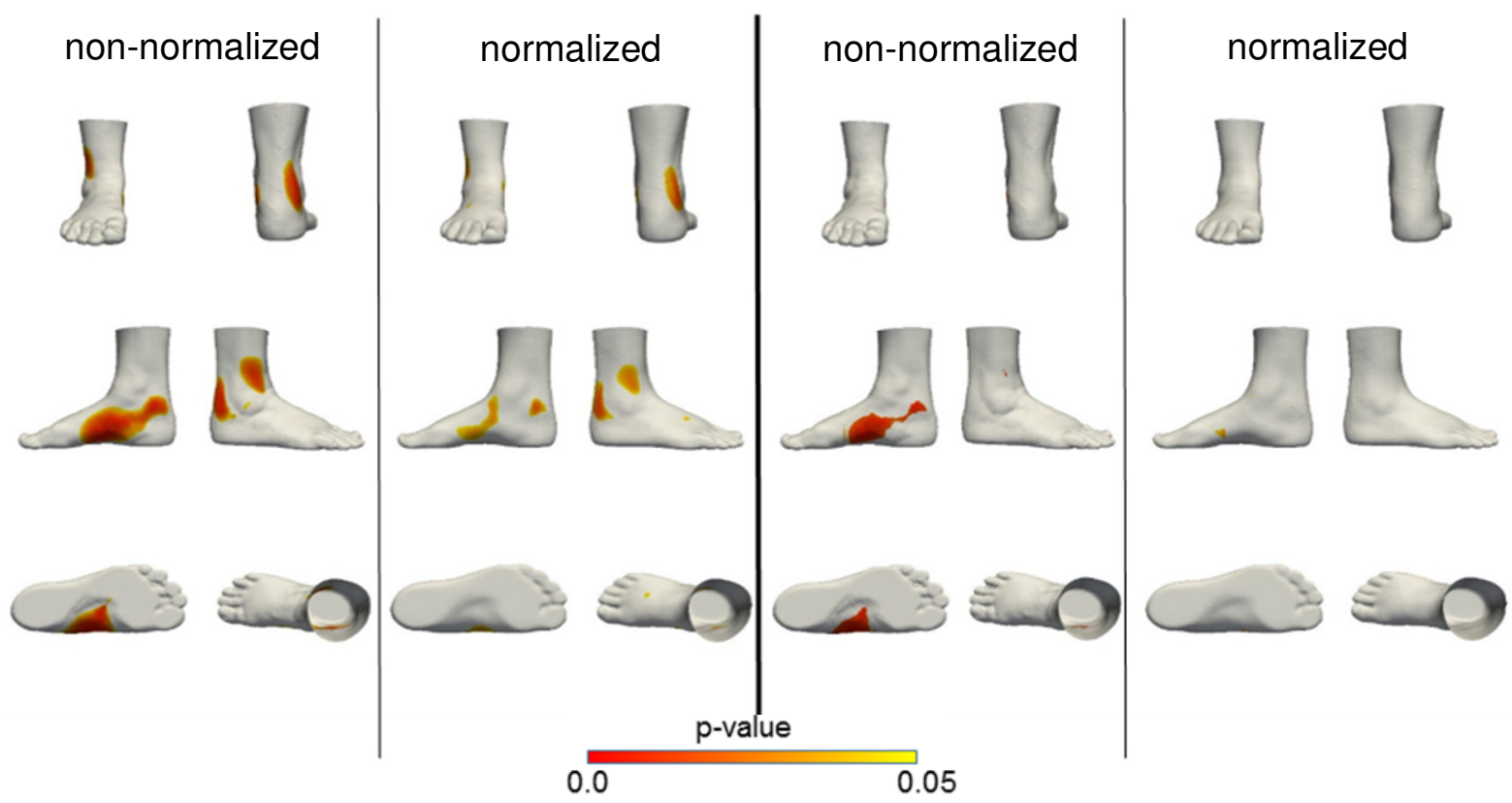

Fig 9. Detected regions for the non-normalized and normalized test feet (hallux valgus, heel spur, pronation) before and after FDR correction. 


\section{Pronation}

\section{uncorrected}

\section{FDR correction}

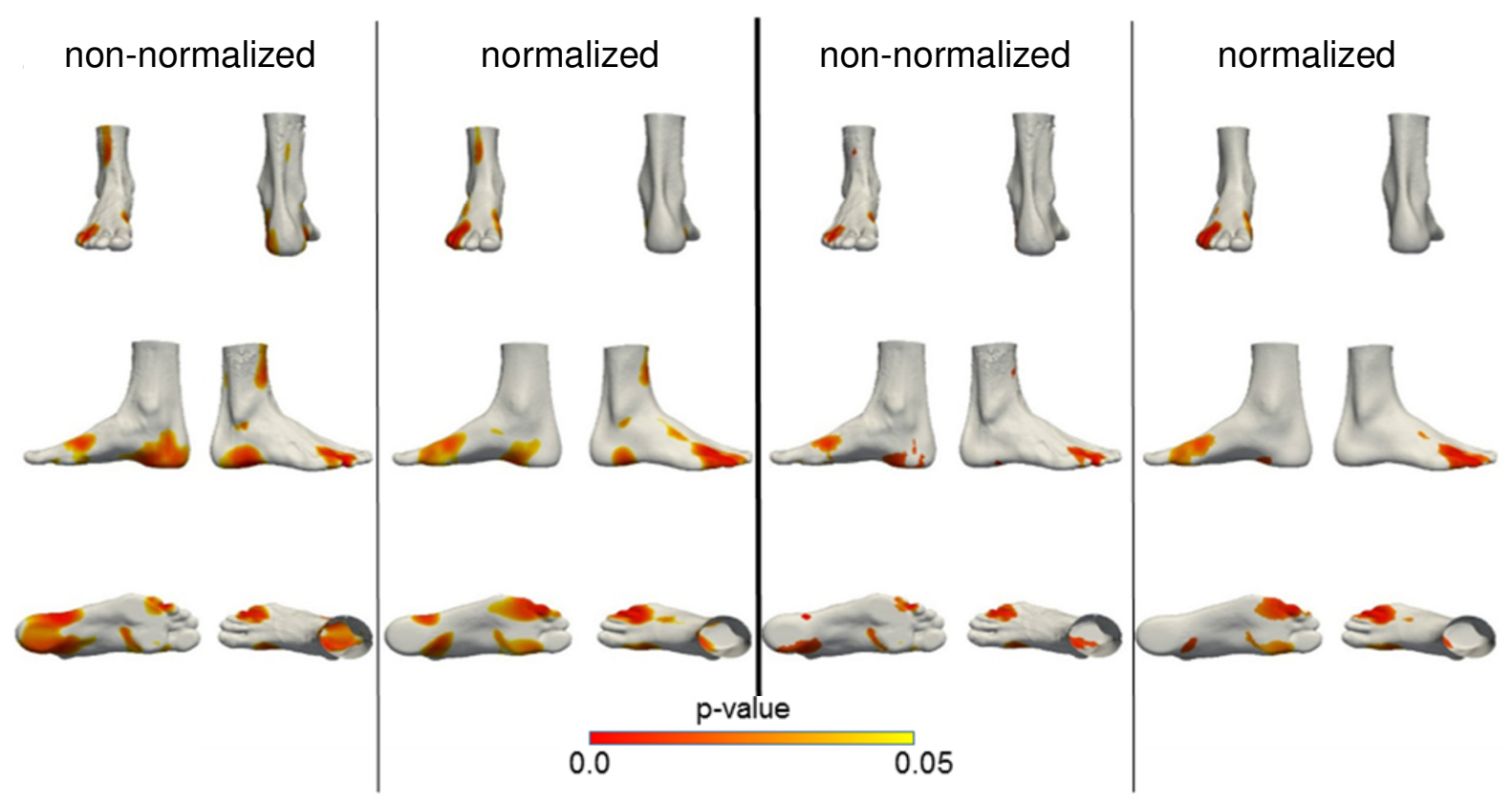

Fig 9. (Cont.) Detected regions for the non-normalized and normalized test feet (hallux valgus, heel spur, pronation) before and after FDR correction.

\section{Discussion}

\subsection{General observations}

This study proposed methods that enable the construction of 3D foot models of different foot types. A visual inspection of the obtained normal and flat foot models clearly showed the expected difference in foot curve on the average surface.

Big abnormal regions of the foot with heel spur and the foot with pronation were detected due to large deviations with respect to the 3D foot model. The foot with heel spur has a wider region around the heel (tarsal bones) that is visible as an extreme in comparison to the feet that are within the 3D foot model. The vertices of the midfoot surface of the foot with heel spur are slightly moved towards the toes tangentially along the surface causing that points from that region become extreme and are therefore detected as an abnormality (Fig. 10). The underlying reason for this tangential shift will be the subject of future research where we will try to rule out registration artifacts as a possible cause.

The foot with pronation is more narrow than the other feet within the 3D foot model and has a high arch. Due to the choice of the least square alignment, regions at the rear foot and forefoot of the foot with pronation are detected as abnormal. Different alignments, e.g. using the ground plane as a reference, should be evaluated in the future for the more intuitive detection of the arch as the abnormal region (Fig. 11).

The foot with hallux valgus is approximately similar in size to the 3D foot model. Extreme deviations for this test foot are in the toe region and most notably in the first metatarsal head (connected to the big toe), they are therefore detected as abnormal regions. The effect of normalization for the foot with hallux valgus revealed an additional abnormal regions in the distal toe area (Fig 9. first row) what could be expected for this pathology considering the moved biggest toe effects also the other toes. 


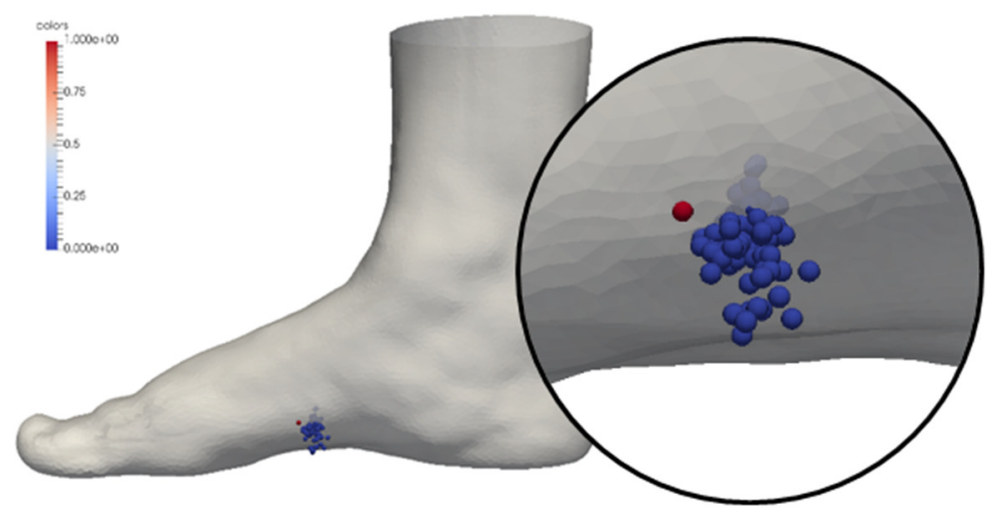

Fig 10. Red point of the foot with heel spur is an extreme (tangential direction) compared to the corresponding points within foot model (blue points)

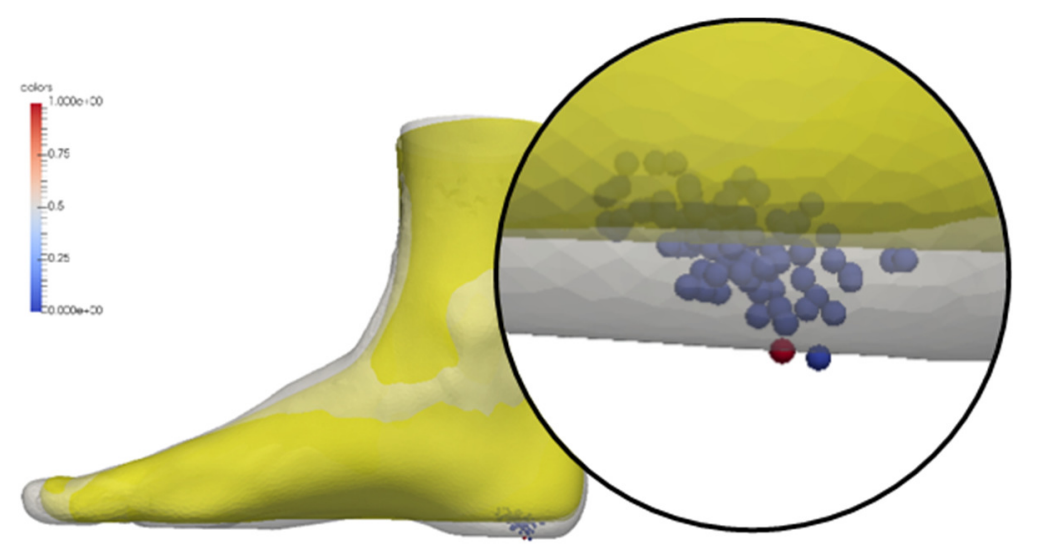

Fig 11. Overlapped foot model (yellow) and foot with pronation (white) show that alignment to the ground plane would be more intuitive for detecting arch as abnormal region

\subsection{Influence of normalization}

To decrease the influence of foot size on 3D foot model building and abnormality detection, different alignment transformations were evaluated: rigid transformation resulting in a non-normalized model and similarity transformations resulting in a normalized model. After comparing the detected regions of the normalized and non-normalized version for each test foot, we obtained following results. For the foot with heel spur, larger regions of abnormality were detected in the non-normalized case, $11.61 \%$ of the foot surface area versus $5.16 \%$ in the normalized case. The bigger regions are due to the size difference between the test foot and the model foot. Also larger regions were detected for the foot with pronation: $12.31 \%$ non-normalized compared to $5.09 \%$ normalized. As the foot with pronation is more narrow than the model foot, bigger regions of abnormality are expected in the non-normalized case due to the fact the used alignment doesn't remove scaling differences. The foot with hallux valgus didn't show this trend of decreasing the size of abnormality regions from normalized to nonnormalized testing. For the normalized foot with hallux valgus, the size of the detected regions increased from $3.00 \%$ to $4.41 \%$ of the whole foot.

The bigger regions were detected for non-normalized foot with heel spur and the foot with pronation due to the smaller size of both feet comparing to the 3D foot model. The normalization removes the scale effect and as a result detected regions after normalization are more accurately mapped. We therefore give preference to the procedure including normalization as it is independent of foot size.

\subsection{Correction for multiple comparisons}

To decrease effects of false outliers we performed FDR correction. Within this procedure false outliers were rejected and abnormal regions were more accurately mapped. After the FDR correction, we obtained results with smaller detected regions of abnormalities, what was expected. For the normalized foot with hallux valgus detected regions decreased from $4.41 \%$ to $2.76 \%$. The detected 
regions in forefoot and toe area decreased in size after FDR correction, but also the region in the rear foot that was detected using non-normalized model disappeared after FDR correction (Fig. 9 first row) due to the fact it is not such a strong outlier. The normalization of the foot with heel spur has also resulted in decreasing the abnormal regions from $5.16 \%$ to $0.19 \%$. Using this procedure abnormal regions weren't detected for the foot with heel spur, which is not surprising since a heel spur pathology is usually not visible from a 3D foot scan (Fig 9. second row) and requires a dynamic plantar pressure measurement. The trend of decreasing detected regions of abnormality is also observed for the foot with pronation from $16.77 \%$ to $9.72 \%$. Weaker outliers of the foot with pronation caused the reduction of the size of detected regions of abnormality.

\section{Conclusion}

In this paper 3D models for normal arched feet and flat feet were built. Also, a method was proposed that enables the detection of different abnormalities of previously unseen feet. The accuracy of the pathology detection can be increased by increasing the size of the database. For future work, the authors will evaluate the influence of separating normal from tangential deviations in the statistical testing. In addition, to obtain more specific models for abnormality testing, patient factors, such as gender, shoe size and age, will be included in the 3D foot model, potentially resulting in even more accurate pathology detection. Finally, different alignments will be considered, such as alignment to the ground plane as opposed to the least square alignment, which might result in a more intuitive detection of abnormal regions.

\section{References}

[1] H. Jonely, J.-M. Brismee, P. S. Sizer Jr., C. R. James, Relationships between clinical measures of static foot posture and plantar pressure during static standing and walking, Clinical Biomechanics 26 (8) (2011) 873-879.

[2] S. Telfer et al. "The use of 3D surface scanning measurement and assessment of the human foot" J Foot Ankle Res, vol5, 2010

[3] T. C. Pataky, J. Y. Goulermas, Pedobarographic statistical parametric mapping (pSPM): A pixellevel approach to foot pressure image analysis, Journal of Biomechanics 41 (2008) 2136-2143.

[4] A. D. Cock, J. Vanrenterghem, T. Willems, E. Witvrouw, D. D. Clercq, The trajectory of the centre of pressure during barefoot running as a potential measure for foot function, Gait \& Posture 27 (4) (2008) 669-675.

[5] Jee, Soo-chan, Sanghyun Kwon, and Myung Hwan Yun. "Analysis of stature prediction from foot anthropometry: a South Korean case study." Australian Journal of Forensic Sciences (2015): 113.

[6] Price, Carina, and Christopher Nester. "Foot dimensions and morphology in healthy weight, overweight and obese males." Clinical Biomechanics 37 (2016): 125-130.

[7] Kim, Nam-Soon, and Wol-Hee Do. "Classification of Elderly Women's Foot Type." Journal of the Korean Society of Clothing and Textiles 38.3 (2014): 305-320.

[8] Yu-Chi Lee \& Mao-Jiun Wang (2015) "Taiwanese adult foot shape classification using 3D scanning data", Ergonomics, 58:3, 513-523

[9] J. Shiraishi, Q. Li, D. Appelbaum, and K. Doi, Computer-Aided Diagnosis and Artificial Intelligence in Clinical Imaging, Seminars in Nuclear Medicine, 41 (6) (2011) 449-462.

[10] B. G. Booth, S. P. Miller, C. J. Brown, K. J. Poskitt, V. Chau, R. E. Grunau, A. R. Synnes, G. Hamarneh, STEAM - statistical template estimation for abnormality mapping: A personalized DTI analysis technique with applications to the screening of preterm infants, Neurolmage 125 (2016) 705-723.

[11] Brian Amberg, Sami Romdhani, and Thomas Vetter. Optimal step nonrigid ICP algorithms for surface registration. CVPR'07, June 2007

[12] F. Danckaers et al. "Correspondence Preserving Elastic Surface Registration with Shape Model Prior", International conference of Pattern Recognition, 2014

[13] F. Danckaers et al. "Evaluation of 3D Body Shape Predistions Based on Features", International Conference on 3D Body Scanning Technologies, 2015, doi:10.15221/15.258

[14] MeshLab, Visual Computing Lab - ISTI - CNR, http://meshlab.sourceforge.net/

[15] Gower, John C. "Generalized procrustes analysis." Psychometrika 40.1 (1975): 33-51.

[16] FOOTWORK: Automated Digital Process for Higher Orthotics Quality, iMinds ICON project 20152017, https://www.iminds.be/en/projects/FOOTWORK 